\title{
Poisoning by toxic plants in Hong Kong: a 15-year review
}

\author{
WY Ng, LY Hung, YH Lam, SS Chan, KS Pang, YK Chong, CK Ching, Tony WL Mak*
}

\section{A B S T R A C T}

Introduction: Hong Kong has a great diversity of plants, many of which are toxic to humans. The aim of this study was to identify the plant species most commonly involved in cases of plant poisoning in Hong Kong and to provide clinicians with a reference tool for the diagnosis and management of plant poisoning.

Methods: We retrospectively reviewed all plant poisoning cases referred to the Hospital Authority Toxicology Reference Laboratory from 1 January 2003 to 31 December 2017. Demographics, clinical presentation, laboratory findings, treatment and outcomes of patients, as well as morphological identification and analytical testing of the plant specimens, were investigated.

Results: A total of 62 cases involving 26 poisonous plant species were identified, among which Alocasia macrorrhizos (Giant Alocasia), Gelsemium elegans (Graceful Jessamine), and Rhododendron (Azalea) species were the three most commonly encountered. Gastrointestinal toxicity $(\mathrm{n}=30,48 \%)$, neurological toxicity $(n=22,35 \%)$, and hepatotoxicity $(n=6,10 \%)$ were the three most common clinical problems. Forty-nine (79\%) and eight (13\%) patients had mild and moderate toxicity, respectively; they all

This article was published on $10 \mathrm{Apr}$ 2019 at www.hkmj.org. recovered shortly with supportive treatment. The remaining five (8\%) patients experienced severe toxicity requiring intensive care support. Most patients ( $n=61,98 \%)$ used the plants intentionally: as a medicinal herb $(n=31)$, as food $(n=29)$, and for attempting suicide $(n=1)$. Reasons for using the poisonous plants included misidentification $(n=34$, $55 \%)$, unawareness of the toxicity $(n=20,32 \%)$, and contamination $(n=6,10 \%)$.

Conclusions: Although most plant exposure resulted in a self-limiting disease, severe poisonings were encountered. Epidemiology of plant poisonings is geographically specific. Clinicians should be aware of local poisonous plants and their toxicities.

\section{Hong Kong Med J 2019;25:102-12 \\ https://doi.org/10.12809/hkmj187745}

\author{
WY Ng, MB, ChB, PhD \\ ${ }^{1}$ LY Hung, MB, BS \\ ${ }^{1}$ YH Lam, MPhil \\ ${ }^{1}$ SS Chan, MSc \\ ${ }^{2}$ KS Pang, MSc \\ ${ }^{1}$ YK Chong, FHKAM (Pathology) \\ CK Ching, FRCPA, FHKAM (Pathology) \\ ${ }^{1}$ TWL Mak *, FRCPath, FHKAM (Pathology)
}

${ }^{1}$ Hospital Authority Toxicology Reference Laboratory, Department of Pathology, Princess Margaret Hospital, Laichikok, Hong Kong 2 Hong Kong Herbarium, Agriculture, Fisheries and Conservation Department, Hong Kong

* Corresponding author: makwl@ha.org.hk

New knowledge added by this stud

- The three most common plants causing poisoning in Hong Kong were Alocasia macrorrhizos, Gelsemium elegans, and Rhododendron species.

- Plants causing severe and potentially fatal poisonings in Hong Kong were Abrus precatorius, Gelsemium elegans, Rhododendron species, and Emilia sonchifolia.

Implications for clinical practice or policy

- Plant poisoning is uncommon but can be severe.

- Raising public awareness minimises unintentional poisoning.

- This study provided useful reference for the local clinicians to diagnose and manage plant poisonings.

\section{Introduction}

Many plants are poisonous to humans. Surveys of various toxicology centres in Australia, ${ }^{1}$ Germany, ${ }^{2,3}$ Morocco, ${ }^{4}$ New Zealand, ${ }^{5}$ Sweden, ${ }^{6}$ Thailand, ${ }^{7}$ the United Kingdom, ${ }^{8}$ and the United States ${ }^{9,10}$ showed that plant exposure was responsible for $1.8 \%$ to $8 \%$ of all inquiries. Most plant exposures did not result in significant toxicity, but severe and life-threatening poisonings have been reported. As reported by the Moroccan Poison Control Centre from 1980 to 2011, plants were the cause of approximately $5 \%$ of all fatal cases of intoxication encountered. ${ }^{4}$ Consumption of wild plants as "medicinal herbs" or food is not an uncommon practice in Hong Kong, and severe plant poisoning cases have been reported. ${ }^{11-19}$ However, local epidemiology data on plant poisoning are 
sparse and limited.

Owing to the variable clinical features and rare occurrence of plant poisonings, diagnosing and treating these patients remain a challenge to our frontline clinicians. Although history of plant exposure is important, it is often insufficient to pinpoint the incriminating toxic plant. Misidentification of plant is a common cause of poisoning in the first place. Morphological identification of a poisonous plant and biochemical confirmation are generally not available to guide immediate clinical management. Therefore, clinical features are critical for initiating supportive treatment, which is a common strategy for treating poisonings of an uncertain nature.

Classifications of clinically significant plant toxins have been proposed in order to aid rapid recognition and management of these patients..$^{20,21}$ Plant toxins can be broadly classified into four groups: (1) cardiotoxic toxins, such as cardiac glycosides; (2) neurotoxic toxins, such as gelsemium alkaloids, grayanotoxins, solanaceous tropane (anticholinergic) alkaloids, strychnine, and brucine; (3) cytotoxic toxins, such as colchicine, mimosine, plumbagin, toxalbumins; vinblastine, and vincristine; and (4) gastrointestinal-hepatotoxic toxins, such as amaryllidaceous alkaloids, calcium oxalate raphide, cycasin, pentacyclic triterpenoids, phorbol esters, phytolaccatoxins, plumericin, pyrrolizidine alkaloids, saponins, steroidal alkaloids, tetrahydropalmatine, and teucvin.

The Hospital Authority Toxicology Reference Laboratory (HATRL), the only tertiary clinical toxicology laboratory in Hong Kong, provides support to all local hospitals in managing patients with complex poisoning problem, including cases of plant poisonings. Besides plant identification, HATRL provides specialised toxicology testing for certain plant toxins, especially for those with significant clinical toxicity and management impact. ${ }^{16,22}$ The aim of the present study was to identify the plant species most commonly involved in cases of plant poisoning in Hong Kong, in order to promote awareness among local clinicians and to provide a reference for diagnosing and managing plant poisonings.

\section{Methods}

All cases of suspected plant-related poisoning referred to the HATRL from January 2003 to December 2017 were retrospectively reviewed. Clinical data were collected by reviewing the laboratory database and patient medical records. Demographic characteristics, clinical presentation, drug history, laboratory and toxicological findings, progress, and outcomes of these patients were reviewed. The causal relationships between the clinical presentation and plant use were evaluated

\section{香港植物中毒個案：十五年病例回顧 \\ 吳煒欣、孔令妍、林英豪、陳淑珊、彭權森、張耀君、 程楚君、來永禮}

引言：香港擁有豐富的植物資源, 在我們數以千計的植物品種當中, 部分含有毒性。本研究旨在探討香港常見之植物中毒案例, 並為日後 的相關中毒個案提供臨床診斷及治療方向。

方法：這項回顧性研究分析2003年1月1日至2017年12月 31 日期間轉 介至醫院管理局毒理學參考化驗室有關植物中毒的案例, 並整合患者 的人口統計學資料、臨床症狀和相關植物標本的化驗結果及分析其毒 性成份

結果：研究期間共確診 62 病例, 牽涉 26 種有毒植物, 其中最常見的為 海芋、鈎吻和杜鵑。中毒徵狀主要為腸胃毒性 30 例 $(48 \%$ ) 、神經系 統毒性22例（35\%）及肝毒性6例（10\%）。其中49例（79\%）和8例 （13\%）分別屬於輕微和中度中毒, 患者接受症狀治療後完全康復; 其餘5例（8\%）為嚴重中毒個案, 患者需接受深切治療。患者使用有 關植物作藥材佔31例, 食材佔29例, 並有一例用作自殺。有關藥材 / 食材引致中毒的原因源於誤認植物品種34例（55\%）、不了解植物毒 性20例（32\%）及進食被毒素污染的植物6例（10\%）。

結論：在香港發生的植物中毒個案大多為輕微中毒, 嚴重中毒病例亦 偶有發生。本研究綜合本化驗室過去十五年間處理本地植物中毒的經 驗, 可作為醫生臨床診斷及治療的參考工具。

based on known adverse effects of the specified plant or toxins, the temporal sequence, exclusion of other underlying or concurrent diseases which could otherwise account for the clinical presentation, and progress after discontinuation of plant use. Severity of the poisoning cases was graded according to an established poisoning severity score as follows ${ }^{23}$ : mild, transient, and spontaneously resolving symptoms (mild); pronounced or prolonged symptoms (moderate); severe or life-threatening symptoms (severe); and fatal poisoning (fatal). Descriptive statistics were used to present the results.

In collaboration with the Hong Kong Herbarium, Agriculture, Fisheries and Conservation Department, available plant specimens were sent for morphological identification. Mass spectrometrybased and microscopy-based tests for specific plant toxin(s) were developed, and performed on selected cases, guided by clinical presentation of the patients and types of the toxic plant(s) exposed. Mass spectrometry-based analytical platforms can identify the majority of the clinically significant plant toxins affecting the cardiovascular, neurological, gastrointestinal, hepatological, and renal systems in plant and biological specimens. The poisonous plants commonly encountered in Hong Kong and the corresponding plant toxins are summarised in Table $1 .^{24}$

The ethics committee exempted the study group from obtaining patient consent because the presented data were anonymised, and the risk of identification was low. The STROBE guidelines were used to ensure the reporting of this study. ${ }^{25}$ 
TABLE I. Mechanism of toxicity and poisoning features of common plant toxins, and the corresponding poisonous plants in Hong Kong ${ }^{24}$

\begin{tabular}{|c|c|c|c|c|}
\hline Plant toxins & Mechanism of toxicity & $\begin{array}{l}\text { Poisoning features reported in the } \\
\text { literature }\end{array}$ & Genera/species & $\begin{array}{l}\text { Common name(s) } \\
\text { in Chinese }\end{array}$ \\
\hline \multicolumn{5}{|l|}{ Cardiotoxic } \\
\hline $\begin{array}{l}\text { Cardiac } \\
\text { glycosides* }\end{array}$ & $\begin{array}{l}\text { Inhibit } \mathrm{Na}^{+} / \mathrm{K}^{+}-\mathrm{ATP} \text { ase of the } \\
\text { myocardium, increase its } \\
\text { excitability and may lead to } \\
\text { arrhythmia }\end{array}$ & $\begin{array}{l}\text { Mimics digoxin toxicity, nausea, } \\
\text { vomiting, abdominal pain, } \\
\text { arrhythmias, confusion, convulsion }\end{array}$ & $\begin{array}{l}\text { Adenium obesum } \\
\text { Asclepias curassavica } \\
\text { Calotropis gigantea } \\
\text { Catunaregam spinosa } \\
\text { Cerbera manghas } \\
\text { Convallaria majalis } \\
\text { Nerium oleander } \\
\text { Strophanthus divaricatus } \\
\text { Thevetia peruviana }\end{array}$ & $\begin{array}{l}\text { 天寶花 } \\
\text { 馬利筋 } \\
\text { 牛角瓜 } \\
\text { 山石榴 } \\
\text { 海杧果 } \\
\text { 鈴蘭 } \\
\text { 夾竹桃 } \\
\text { 羊角拗 } \\
\text { 黃花夾竹桃 }\end{array}$ \\
\hline
\end{tabular}

\section{Neurotoxic \\ Gelsemium Antagonise acetylcholine

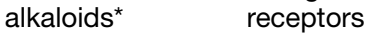

$\begin{array}{ll}\text { Grayanotoxins }^{*} & \begin{array}{l}\text { Prevent inactivation and lead to } \\ \text { persistent opening of voltage- } \\ \text { gated sodium channels }\end{array} \\ \begin{array}{l}\text { Solanaceous } \\ \text { tropane alkaloids }\end{array} & \begin{array}{l}\text { Competitive blockage of } \\ \text { cholinergic muscarinic receptors }\end{array}\end{array}$

\begin{tabular}{ll}
$\begin{array}{l}\text { Strychnine and } \\
\text { brucine* }^{*}\end{array}$ & $\begin{array}{l}\text { Act as glycine receptor antagonist } \\
\text { result in hyperexcitability of spinal } \\
\text { nerves }\end{array}$ \\
$\begin{array}{l}\text { Cytotoxic } \\
\text { related toxins }\end{array}$ & $\begin{array}{l}\text { Prevent assembly of } \\
\text { microtubules, resulting in failure } \\
\text { of mitosis }\end{array}$ \\
\hline $\begin{array}{l}\text { Mimosine* } \\
\text { Inhibit the initiation of DNA } \\
\text { replication }\end{array}$ \\
$\begin{array}{l}\text { Plumbagin } \\
\text { Increase the formation of reaction } \\
\text { oxygen species in cells, leading } \\
\text { to DNA strand breakage and base } \\
\text { damage }\end{array}$ \\
$\begin{array}{l}\text { Toxalbumins } \\
\text { (eg, abrin*, ricin, } \\
\text { curcin) }\end{array}$ & $\begin{array}{l}\text { Inactivate ribosome and inhibit } \\
\text { protein synthesis }\end{array}$ \\
\hline $\begin{array}{l}\text { Vinblastine and } \\
\text { vincristine* }\end{array}$ & $\begin{array}{l}\text { Prevent assembly of } \\
\text { microtubules, resulting in failure } \\
\text { of mitosis }\end{array}$ \\
\hline
\end{tabular}

\section{Gastrointestinal hepatotoxic}

$\begin{array}{ll}\begin{array}{l}\text { Amaryllidaceous } \\ \text { alkaloids }\end{array} & \begin{array}{l}\text { Irritate gastrointestinal tract and } \\ \text { hypotensive effect }\end{array}\end{array}$

Calcium oxalate raphide*
Raphides stored in cells are released when subjected to mechanical pressure such as ingestion and pressure, causing tissue irritation and inflammation
Dry mouth, dizziness, sweating, blurred vision, weakness, hyperthermia, mydriasis, convulsion, respiratory and central nervous system depression

Nausea, vomiting, sweating, dizziness, blurred vision, confusion, arrhythmias, respiratory failure

Anticholinergic toxidrome, including dry mouth, dry skin, flushing, hyperthermia, tachycardia, hypertension, urinary retention, decreased bowel sounds, mydriasis, confusion

Opisthotonos, respiratory paralysis, hyperthermia, rhabdomyolysis, lactic acidosis, renal failure

\section{Gelsemium elegans \\ 鈎吻 / 斷腸草 Gelsemium sempervirens 金鈎吻}

\begin{tabular}{ll} 
Rhododendron species & 杜鵑 \\
& \\
$\begin{array}{l}\text { Solandra nitida } \\
\text { Datura metel }\end{array}$ & 金盃藤 \\
\hline 洋金花 / 白花曼陀羅
\end{tabular}

Nausea, vomiting, diarrhoea, abdominal pain, bone marrow suppression, multi-organ failure

Delayed growth, hair loss, and cataract in animal models

Datura metel

Skin contact: contact dermatitis, stinging pain, blistering Ingestion: vomiting, diarrhoea, miscarriage

Nausea, vomiting, diarrhoea, abdominal pain, gastrointestinal bleeding, tachycardia, drowsiness, weakness, hallucination, convulsion, multi-organ failure

Paraesthesia, stomatitis, paralytic ileus, myalgia, fever, bone marrow suppression, organ failure

\section{Strychnos angustiflora}

牛眼馬錢
Skin contact: contact dermatitis. Ingestion: nausea, vomiting, diarrhoea, perioral numbness dizziness, blurred vision, hypotension, convulsion

Skin contact: itchiness Eye contact: irritation, blindness Ingestion: burning sensation of the oral cavities, salivation, nausea, vomiting, diarrhoea, dysphagia, dysphonia

$\begin{array}{ll}\text { Hymenocallis littoralis } & \text { 水鬼蕉 } \\ \text { Lycoris aurea } & \text { 忽地笑 } \\ \text { Narcissus tazetta var. } & \text { 水仙 }\end{array}$

$\begin{array}{ll}\begin{array}{l}\text { Hemerocallis citrina } \\ \text { Gloriosa superba }\end{array} & \text { 黃花菜 / 金針菜 } \\ \text { Mimosa pudica } & \text { 嘉蘭 } \\ \text { Leucaena leucocephala } & \text { 含羞草 } \\ \text { Plumbago zeylanica } & \text { 銀合歡 } \\ & \text { 白花丹 } \\ & \\ \text { Abrus precatorius } & \\ \text { Ricinus communis } & \text { 相思子 } \\ & \text { 蓖麻 }\end{array}$

Catharanthus roseus 長春花

水鬼蕉
忽地笑
水仙

海芋
尖尾芋
一把傘南星
花葉萬年青
龜背竹
犁頭尖
馬蹄蓮
風信子
水仙

Alocasia macrorrhizos

Alocasia cucullata

Arisaema erubescens

Dieffenbachia seguine

Monstera deliciosa

Typhonium blumei

Zantedeschia aethiopica

Hyacinthus orientalis

Narcissus tazetta var.

chinensis

\footnotetext{
* Specific toxicological investigation available in The Hospital Authority Toxicology Reference Laboratory
} 
TABLE I. (cont'd)

\begin{tabular}{|c|c|c|c|c|}
\hline Plant toxins & Mechanism of toxicity & $\begin{array}{l}\text { Poisoning features reported in the } \\
\text { literature }\end{array}$ & Genera/species & $\begin{array}{l}\text { Common name(s) } \\
\text { in Chinese }\end{array}$ \\
\hline Cycasin & Irritate gastrointestinal tract & $\begin{array}{l}\text { Nausea, vomiting, diarrhoea, } \\
\text { abdominal pain, headache, dizziness }\end{array}$ & Cycas revoluta & 蘇鐵 \\
\hline $\begin{array}{l}\text { Pentacyclic } \\
\text { triterpenoids }\end{array}$ & $\begin{array}{l}\text { May be hepatotoxic and cause } \\
\text { intrahepatic cholestasis }\end{array}$ & $\begin{array}{l}\text { Vomiting, diarrhoea, abdominal pain, } \\
\text { respiratory depression and liver } \\
\text { impairment }\end{array}$ & Lantana camara & 馬縜丹 \\
\hline Phorbol esters & Irritate gastrointestinal tract & $\begin{array}{l}\text { Nausea, vomiting, diarrhoea, } \\
\text { abdominal pain }\end{array}$ & $\begin{array}{l}\text { Aleurites moluccana } \\
\text { Croton tiglium } \\
\text { Vernicia montana }\end{array}$ & $\begin{array}{l}\text { 石栗 } \\
\text { 巴豆 } \\
\text { 木油樹 }\end{array}$ \\
\hline Phytolaccatoxins ${ }^{\star}$ & $\begin{array}{l}\text { Irritants to mucosal surface and } \\
\text { have haemolytic effect }\end{array}$ & $\begin{array}{l}\text { Weakness, nausea, vomiting, } \\
\text { diarrhoea, abdominal pain }\end{array}$ & Phytolacca americana & 美洲商陸 \\
\hline Plumericin & Irritate gastrointestinal tract & $\begin{array}{l}\text { Skin contact: rash, contact dermatitis } \\
\text { Ingestion: vomiting, diarrhoea, } \\
\text { abdominal pain, dehydration }\end{array}$ & $\begin{array}{l}\text { Allamanda cathartica } \\
\text { Allamanda schottii }\end{array}$ & $\begin{array}{l}\text { 軟枝黃蟬 } \\
\text { 黃蟬 }\end{array}$ \\
\hline $\begin{array}{l}\text { Pyrrolizidine } \\
\text { alkaloids* }\end{array}$ & $\begin{array}{l}\text { Convert to highly reactive } \\
\text { metabolites by liver, which bind } \\
\text { to cellular components causing } \\
\text { acute and chronic toxicities }\end{array}$ & $\begin{array}{l}\text { Acute: abdominal pain, } \\
\text { hepatomegaly, ascites, liver necrosis, } \\
\text { death } \\
\text { Chronic: hepatomegaly, recurrent } \\
\text { ascites, hepatic vein occlusion, } \\
\text { cirrhosis, hepatocellular carcinoma, } \\
\text { pulmonary hypertension, death }\end{array}$ & $\begin{array}{l}\text { Crotalaria pallida var. obovata } \\
\text { Crotalaria retusa } \\
\text { Emilia sonchifolia } \\
\text { Farfugium japonicum } \\
\text { Gynura divaricata } \\
\text { Heliotropium indicum } \\
\text { Senecio scandens }\end{array}$ & $\begin{array}{l}\text { 豬屎豆 } \\
\text { 吊裙草 } \\
\text { 一點紅 } \\
\text { 大吳風草 } \\
\text { 白子菜 } \\
\text { 大尾搖 } \\
\text { 千里光 }\end{array}$ \\
\hline Saponins & Irritate gastrointestinal tract & Vomiting, diarrhoea, abdominal pain & $\begin{array}{l}\text { Albizia lebbeck } \\
\text { Aleurites moluccana } \\
\text { Cardiospermum halicacabum } \\
\text { Costus speciosus }\end{array}$ & $\begin{array}{l}\text { 大葉合歡 } \\
\text { 石栗 } \\
\text { 倒地鈴 } \\
\text { 閉鞘踾 }\end{array}$ \\
\hline $\begin{array}{l}\text { Steroidal } \\
\text { alkaloids* }\end{array}$ & $\begin{array}{l}\text { Bind to sterols, disrupt cell } \\
\text { membrane integrity and cause } \\
\text { tissue damage }\end{array}$ & $\begin{array}{l}\text { Nausea, vomiting, diarrhoea, } \\
\text { abdominal pain, dizziness, delirium, } \\
\text { slurring of speech, ataxia }\end{array}$ & $\begin{array}{l}\text { Solanum americanum } \\
\text { Solanum capsicoides } \\
\text { Solanum mammosum }\end{array}$ & $\begin{array}{l}\text { 少花龍腠 } \\
\text { 牛茄子 } \\
\text { 乳茄 / 五代同堂茄 }\end{array}$ \\
\hline $\begin{array}{l}\text { Tetrahydro- } \\
\text { palmatine* }\end{array}$ & Cause apoptosis of hepatocytes & $\begin{array}{l}\text { Fever, nausea, vomiting, diarrhoea, } \\
\text { abdominal pain, jaundice, } \\
\text { hepatomegaly, liver impairment, } \\
\text { drowsiness, weakness and central } \\
\text { nervous system depression }\end{array}$ & Stephania sinica & 汝蘭 \\
\hline Teucvin & $\begin{array}{l}\text { Share similar structure with the } \\
\text { hepatotoxin teucrin A, which can } \\
\text { cause hepatitis through direct } \\
\text { toxicity and secondary immune- } \\
\text { mediated injury }\end{array}$ & Nausea, vomiting, jaundice, hepatitis & Teucrium viscidum & 血見愁 / 山藿香 \\
\hline
\end{tabular}

\section{Results}

A total of 62 cases of confirmed plant poisoning, involving 26 plant species, were identified within the study period. The patients were referred from 14 local hospitals administered by the Hospital Authority. Almost all patients $(\mathrm{n}=60,97 \%)$ were Chinese, 29 (47\%) were male, and 33 (53\%) were female. The median age was 50 years (range, 1 month to 83 years), including three children (range, 1-23 months) and four adolescents (range, 15-18 years). The route of exposure was oral in $60(97 \%)$ patients and topical in two $(3 \%)$ patients. The majority of the patients $(\mathrm{n}=55,89 \%)$ developed acute toxic symptoms after a single use of the plants, while the remaining patients $(n=7,11 \%)$ reported a history of prolonged exposure. Details of the plant poisoning cases, including the involved plant species and toxins, clinical presentation and outcome of these patients are summarised in Table 2.

Gastrointestinal toxicity was the most common clinical presentation $(n=30,48 \%)$. Of these 30 patients, 17 developed oromucosal irritation after ingestion of calcium oxalate raphidecontaining plants. The other 13 patients presented with gastroenteritis-like symptoms, such as nausea, vomiting, abdominal pain and diarrhoea, after ingestion of plant containing gastrointestinal irritants $(n=11)$ or cytotoxic toxins $(n=2)$. Neurological toxicity was the second most common presenting symptoms $(n=22,35 \%)$, mainly caused by gelsemium alkaloids $(n=12)$, Solanaceous tropane alkaloids $(n=4)$, and grayanotoxins $(n=3)$. Hepatotoxicity was encountered in six (10\%) patients. Five patients had abnormal liver function test results owing to the use of teucvin $(n=1)$, pyrrolizidine alkaloids $(n=1)$, and other unknown hepatotoxin $(\mathrm{s})(\mathrm{n}=3)$; one patient developed cholestasis after exposure to pentacyclic triterpenoids. Cases of nephrotoxicity $(\mathrm{n}=2,3 \%)$, cardiotoxicity $(\mathrm{n}=1,2 \%)$, and dermatological toxicity 
TABLE 2. Summary of plant poisoning cases encountered from 2003 to 2017, details and severity of poisonings, presentation signs and symptoms, treatment, and outcome of the patients

\begin{tabular}{lll}
\hline $\begin{array}{l}\text { Poisonous plant } \\
\text { involved }\end{array}$ & Plant toxin(s) & $\begin{array}{l}\text { Details of poisoning } \\
\text { Presenting signs and symptoms } \begin{array}{l}\text { Treatment and outcome of } \\
\text { the patient(s) }\end{array}\end{array}$
\end{tabular}

\section{Gastrointestinal toxicity $(\mathbf{n}=30)$}

Alocasia Calcium oxalate
macrorrhizos, raphide
Alocasia cucullata,
and other raphide-
containing plants
$(\mathrm{n}=17)$

\section{Vernicia montana Phorbol esters $(n=3)$}

$\begin{array}{ll}\text { Justicia } & \text { Unknown } \\ \text { gendarussa }(\mathrm{n}=2) & \begin{array}{l}\text { gastrointestinal } \\ \text { irritant }\end{array}\end{array}$

$\begin{array}{ll}\begin{array}{l}\text { Sauropus } \\ \text { spatulifolius }(\mathrm{n}=2)\end{array} & \begin{array}{l}\text { Unknown } \\ \text { gastrointestinal } \\ \text { irritant }\end{array} \\ \begin{array}{l}\text { Phytolacca } \\ \text { americana }(\mathrm{n}=1)\end{array} & \text { Phytolaccatoxin }\end{array}$

$\begin{array}{lll}\begin{array}{l}\text { Solanum } \\ \text { americanum }(\mathrm{n}=1)\end{array} & \begin{array}{l}\text { Steroidal alkaloid } \\ \text { Vernonia }\end{array} & \begin{array}{l}\text { - Took the leaves and fruits of the } \\ \text { plant as wild vegetable }\end{array} \\ \begin{array}{ll}\text { Umygnown } \\ \text { amylina }(\mathrm{n}=1)\end{array} & \begin{array}{l}\text { gastrointestinal } \\ \text { irritant }\end{array} & \begin{array}{l}\text { Took fresh leaves of the plant for } \\ \text { medicinal purposes }\end{array}\end{array}$

$\begin{array}{ll}\begin{array}{l}\text { Cycas revoluta } \\ (n=1)\end{array} & \text { Cycasin } \\ \text { fruit/nut }\end{array}$

\section{Hemerocallis Colchicine \\ citrina $(\mathrm{n}=1)$}

Abrus precatorius Toxalbumins $(n=1)$

\section{Neurotoxicity ( $\mathbf{n = 2 2 )}$}

$\begin{array}{ll}\text { Gelsemium } & \text { Gelsemium } \\ \text { elegans }(n=12) & \text { alkaloids }\end{array}$

Datura metel and Solanaceous other solanaceous tropane alkaloids tropane alkaloidcontaining plants $(n=4)$
- Ingested root of the plant due to misidentification as wild taro $(n=9)$

- Ingested other parts of the plants due to misidentification as edible vegetable $(n=7)$

- Accidentally bit on a leaf of unknown plant species $(n=1)^{*}$

- Took kernels of its fruit due to misidentification as edible nuts $(\mathrm{n}=3$, in a cluster)

- Took herbal broth prepared with the plant for medicinal purpose $(n=1)$

- Took herbal broth prepared with the plant due to misidentification as medicinal plant $(n=1)$

- Took soup prepared with leaves or root of the plant $(n=2)$

- Took herbal broth prepared with root of the plant due to misidentification as "fresh ginseng"

\section{- Took cooked fresh flower of the plant as vegetable}

- Took herbal broth prepared with stem, leaves and seed of the plant for 10 days due to misidentification as "touch-me-not"
Mild toxicity $(n=17)$ : lip and tongue All recovered within a day swelling $(n=11)$, burning sensation with supportive management and pain in oral cavity $(n=11)$, tongue numbness $(n=6)$, sore throat $(n=1)$

Mild toxicity $(n=3)$ : vomiting $(n=3)$, All recovered within a day abdominal pain $(n=3)$ with supportive management

Mild toxicity $(n=2)$ : dizziness $(n=2)$, All recovered within a day vomiting $(n=2)$, diarrhoea $(n=1)$ with supportive management

Mild toxicity $(n=2)$ : vomiting $(n=2), \quad$ All recovered within a day diarrhoea $(n=2)$, abdominal pain with supportive management $(\mathrm{n}=1)$

Mild toxicity $(n=1)$ : nausea, repeated vomiting

Recovered within a day with supportive management

Mild toxicity $(n=1)$ : nausea, dizziness, repeated vomiting

Recovered within a day with supportive management

Mild toxicity $(n=1)$ : vomiting, dizziness

Recovered within a day with supportive management

Mild toxicity $(n=1)$ : abdominal pain, vomiting, dizziness, lip numbness, diarrhoea

Recovered within a day with supportive management

Mild toxicity $(n=1)$ : vomiting, diarrhoea

Severe toxicity $(n=1)$ : nausea, vomiting, acute renal failure

Recovered within a day with supportive management

Required haemodialysis and intensive care support for several weeks

All recovered within a day with supportive management $(n=10)$, blurred vision $(n=5)$ weakness $(n=3)$, vomiting $(n=2)$ the plant due to misidentification as non-poisonous plant $(n=4$, in a cluster)

- Took herbal broth prepared with non-poisonous plant which was contaminated with gelsemium alkaloids ( $n=5$, in two clusters)

- Took herbal broth prepared with the plant for suicidal attempt $(n=1)$

- Took herbal broth prepared with non-poisonous plant which was contaminated with Gelsemium species $(n=1)$

- Accidentally took herbal wine selfprepared with the plant which was intended for topical use $(n=1)$

- Took herbal tea prepared with the plant due to misidentification as non-poisonous species $(n=1)$

- Took root of tropane alkaloidcontaining plants as edible species $(\mathrm{n}=3$, in a cluster)
Severe toxicity $(n=2)$ : respiratory depression $(n=2)$, confusion $(n=1)$, coma $(n=1)$

Required intubation and intensive care support for days, fully recovered

Mild toxicity $(n=4)$ : confusion $(n=4)$, dizziness $(n=3)$, nausea $(n=3)$, vomiting $(n=3)$
All recovered within a day with supportive management 
TABLE 2. (cont'd)

\begin{tabular}{|c|c|c|c|c|}
\hline $\begin{array}{l}\text { Poisonous plant } \\
\text { involved }\end{array}$ & Plant toxin(s) & Details of poisoning & $\begin{array}{l}\text { Presenting signs and symptoms } \\
\text { and severity of poisoning }\end{array}$ & $\begin{array}{l}\text { Treatment and outcome of } \\
\text { the patient(s) }\end{array}$ \\
\hline \multirow[t]{3}{*}{$\begin{array}{l}\text { Rhododendron } \\
\text { species }(n=3)\end{array}$} & Grayanotoxins & $\begin{array}{l}\text { - Took pieces of petals of its flower } \\
\text { for medicinal purpose }(n=1)\end{array}$ & $\begin{array}{l}\text { Mild toxicity }(n=1) \text { : facial } \\
\text { numbness, blurred vision }\end{array}$ & $\begin{array}{l}\text { Recovered within a day with } \\
\text { supportive management }\end{array}$ \\
\hline & & $\begin{array}{l}\text { Took } 10 \text { flowers due to } \\
\text { misidentification as non-poisonous } \\
\text { species }(n=1)\end{array}$ & $\begin{array}{l}\text { Moderate toxicity }(\mathrm{n}=1) \text { : nausea, } \\
\text { vomiting, blurred vision, muscle } \\
\text { cramps, bradycardia, hypotension }\end{array}$ & $\begin{array}{l}\text { Required IV atropine and IVF, } \\
\text { recovered with supportive } \\
\text { treatment in few days }\end{array}$ \\
\hline & & $\begin{array}{l}\text { Took herbal broth prepared with } \\
\text { the flower due to misidentification } \\
\text { as non-poisonous species }\left(n=1^{\star}\right)\end{array}$ & $\begin{array}{l}\text { Severe toxicity }(n=1) \text { : excessive } \\
\text { salivation, vomiting, cyanosis, } \\
\text { limb twitching, bradycardia, } \\
\text { respiratory depression }\end{array}$ & $\begin{array}{l}\text { Required intubation and } \\
\text { intensive care support for } \\
\text { days, fully recovered and } \\
\text { discharged after } 1 \text { week }\end{array}$ \\
\hline $\begin{array}{l}\text { Mirabilis jalapa } \\
(\mathrm{n}=1)\end{array}$ & $\begin{array}{l}\text { Unknown } \\
\text { neurotoxin }\end{array}$ & $\begin{array}{l}\text { Took herbal soup prepared with } \\
\text { root of the plant for medicinal } \\
\text { purpose }\end{array}$ & $\begin{array}{l}\text { Mild toxicity }(n=1) \text { : dizziness, } \\
\text { headache, muscle weakness }\end{array}$ & $\begin{array}{l}\text { Recovered within a day with } \\
\text { supportive management }\end{array}$ \\
\hline $\begin{array}{l}\text { Anredera cordifolia } \\
(n=1)\end{array}$ & $\begin{array}{l}\text { Unknown } \\
\text { neurotoxin }\end{array}$ & $\begin{array}{l}\text { - Took herbal tea prepared with } \\
\text { leaves of the plant due to } \\
\text { misidentification as medicinal plant }\end{array}$ & $\begin{array}{l}\text { Mild toxicity }(n=1) \text { : weakness, limb } \\
\text { numbness }\end{array}$ & $\begin{array}{l}\text { Recovered within a day with } \\
\text { supportive management }\end{array}$ \\
\hline Basella alba $(n=1)$ & $\begin{array}{l}\text { Unknown } \\
\text { neurotoxin }\end{array}$ & $\begin{array}{l}\text { - Took raw stem and fruits of the } \\
\text { plant as vegetable }\end{array}$ & $\begin{array}{l}\text { Mild toxicity }(n=1) \text { : vomiting, limb } \\
\text { numbness, palpitation }\end{array}$ & $\begin{array}{l}\text { Recovered within a day with } \\
\text { supportive management }\end{array}$ \\
\hline \multicolumn{5}{|c|}{ Hepatotoxicity $(n=6)$} \\
\hline $\begin{array}{l}\text { Teucrium viscidum } \\
(\mathrm{n}=1)\end{array}$ & Teucvin & $\begin{array}{l}\text { - Took herbal broth prepared with } \\
\text { the plant for medicinal use for } 1 \\
\text { week }\end{array}$ & $\begin{array}{l}\text { Moderate toxicity }(n=1) \text { : liver } \\
\text { derangement }\end{array}$ & $\begin{array}{l}\text { LFT normalised in few weeks } \\
\text { after discontinuation of the } \\
\text { plant use }\end{array}$ \\
\hline $\begin{array}{l}\text { Lantana camara } \\
(\mathrm{n}=1)\end{array}$ & $\begin{array}{l}\text { Pentacyclic } \\
\text { triterpenoids }\end{array}$ & $\begin{array}{l}\text { - Took herbal broth prepared with } \\
\text { the plant for medicinal purpose for } \\
\text { few weeks }\end{array}$ & $\begin{array}{l}\text { Moderate toxicity }(n=1) \text { : liver } \\
\text { derangement }\end{array}$ & $\begin{array}{l}\text { LFT normalised in few weeks } \\
\text { after discontinuation of the } \\
\text { plant use }\end{array}$ \\
\hline $\begin{array}{l}\text { Juniperus species } \\
(n=1)\end{array}$ & $\begin{array}{l}\text { Unknown } \\
\text { hepatotoxin }\end{array}$ & $\begin{array}{l}\text { - Took herbal tea prepared with } \\
\text { "pine needles" for medicinal } \\
\text { purpose for } 3 \text { weeks }\end{array}$ & $\begin{array}{l}\text { Moderate toxicity }(n=1) \text { : liver } \\
\text { derangement }\end{array}$ & $\begin{array}{l}\text { LFT normalised in few weeks } \\
\text { after discontinuation of the } \\
\text { plant use }\end{array}$ \\
\hline $\begin{array}{l}\text { Blumea } \\
\text { balsamifera }(n=1)\end{array}$ & $\begin{array}{l}\text { Unknown } \\
\text { hepatotoxin }\end{array}$ & $\begin{array}{l}\text { Took herbal tea prepared with the } \\
\text { plant for medicinal purpose for } 1 \\
\text { month }\end{array}$ & $\begin{array}{l}\text { Moderate toxicity }(n=1) \text { : liver } \\
\text { derangement }\end{array}$ & $\begin{array}{l}\text { LFT normalised in few weeks } \\
\text { after discontinuation of the } \\
\text { plant use }\end{array}$ \\
\hline $\begin{array}{l}\text { Emilia sonchifolia } \\
(\mathrm{n}=1)\end{array}$ & $\begin{array}{l}\text { Pyrrolizidine } \\
\text { alkaloids }\end{array}$ & $\begin{array}{l}\text { Took herbal broth prepared with } \\
\text { the plant for medicinal purpose for } \\
6 \text { months }^{*}\end{array}$ & $\begin{array}{l}\text { Severe toxicity }(n=1) \text { : liver } \\
\text { impairment with hepatic vein } \\
\text { thrombosis }\end{array}$ & $\begin{array}{l}\text { Budd-Chiari syndrome with } \\
\text { ascites, pleural effusion and } \\
\text { hepatomegaly requiring } \\
\text { weeks of intensive care unit } \\
\text { support. Resolved in few } \\
\text { months after discontinuation } \\
\text { of the plant use }\end{array}$ \\
\hline $\begin{array}{l}\text { Mimosa pudica } \\
(\mathrm{n}=1)\end{array}$ & Mimosine & $\begin{array}{l}\text { Took herbal broth prepared with } \\
\text { the plant for medicinal purpose for } \\
1 \text { day }\end{array}$ & $\begin{array}{l}\text { Moderate toxicity }(n=1) \text { : liver } \\
\text { derangement }\end{array}$ & $\begin{array}{l}\text { LFT normalised in few weeks } \\
\text { after discontinuation of the } \\
\text { plant use }\end{array}$ \\
\hline \multicolumn{5}{|c|}{ Nephrotoxicity $(n=2)$} \\
\hline $\begin{array}{l}\text { Mimosa pudica } \\
(\mathrm{n}=1)\end{array}$ & Mimosine & $\begin{array}{l}\text { Took herbal broth prepared with } \\
\text { the plant for medicinal purpose } \\
\text { on-and-off for } 1 \text { year }\end{array}$ & $\begin{array}{l}\text { Moderate toxicity }(n=1) \text { : renal } \\
\text { impairment, haematuria, } \\
\text { tubulonephritis confirmed by renal } \\
\text { biopsy }\end{array}$ & $\begin{array}{l}\text { Residual mild renal } \\
\text { impairment after } \\
\text { discontinuation of the plant } \\
\text { use }\end{array}$ \\
\hline $\begin{array}{l}\text { Alternanthera } \\
\text { species }(n=1)\end{array}$ & $\begin{array}{l}\text { Unknown } \\
\text { nephrotoxin }\end{array}$ & $\begin{array}{l}\text { Took herbal broth prepared with } \\
\text { the plant for medicinal purpose for } \\
1 \text { month }\end{array}$ & $\begin{array}{l}\text { Moderate toxicity }(n=1) \text { : acute } \\
\text { kidney injury }\end{array}$ & $\begin{array}{l}\text { Progressed to chronic } \\
\text { kidney disease despite } \\
\text { discontinuation of the plant } \\
\text { use }\end{array}$ \\
\hline \multicolumn{5}{|l|}{ Cardiotoxicity $(n=1)$} \\
\hline $\begin{array}{l}\text { Strophanthus } \\
\text { divaricatus }(n=1)\end{array}$ & Cardiac glycosides & $\begin{array}{l}\text { Topical use of herbal wine } \\
\text { prepared with the skin of the fruit } \\
\text { of the plant for medicinal purpose }\end{array}$ & $\begin{array}{l}\text { Mild toxicity }(n=1) \text { : palpitation, } \\
\text { dizziness }\end{array}$ & $\begin{array}{l}\text { Recovered within a day with } \\
\text { supportive management }\end{array}$ \\
\hline \multicolumn{5}{|c|}{ Dermatological toxicity $(n=1)$} \\
\hline $\begin{array}{l}\text { Plumbago } \\
\text { zeylanica }(n=1)\end{array}$ & Plumbagin & $\begin{array}{l}\text { - Topical use of herbal paste } \\
\text { prepared with the plant due to } \\
\text { misidentification as a medicinal } \\
\text { plant }\end{array}$ & $\begin{array}{l}\text { Mild toxicity }(n=1) \text { : stinging pain, } \\
\text { skin discoloration }\end{array}$ & $\begin{array}{l}\text { Recovered with supportive } \\
\text { management }\end{array}$ \\
\hline
\end{tabular}


(a) Abrus precatorius（相思子）: flower (left), seed (middle), leaf (right)
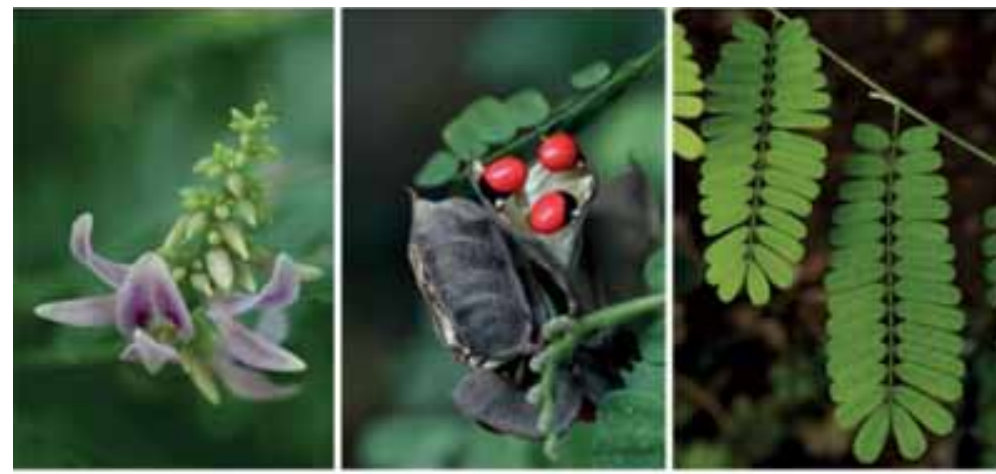

(b) Gelsemium elegans（鈎吻）: flower (left), fruit (middle), leaf (right)
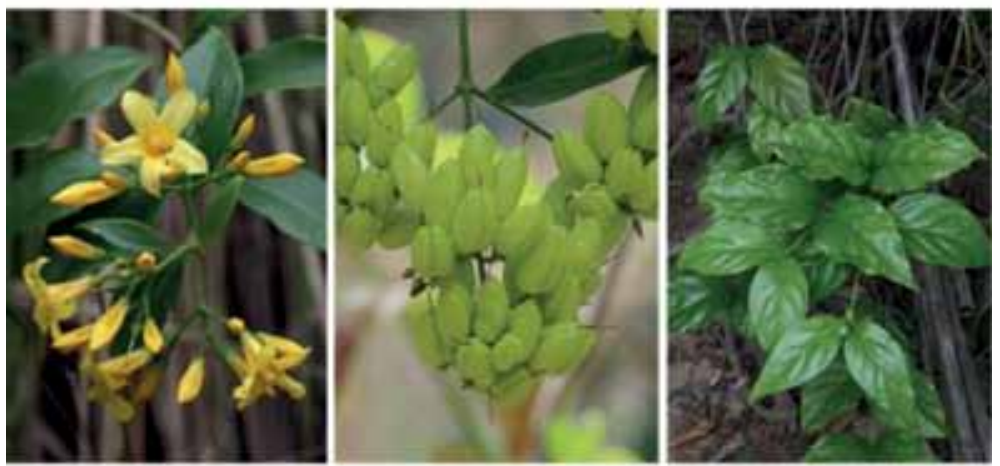

(c) Rhododendron simsii ( 紅杜鵑 ) : flower (left), leaf (middle), whole plant (right)

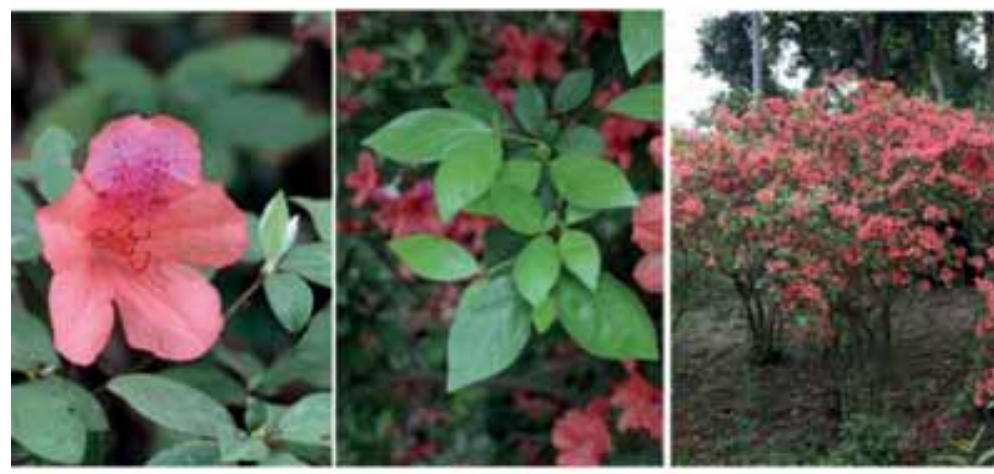

(d) Emilia sonchifolia ( 一點紅 ) : flower (left), leaf (middle), whole plant (right)

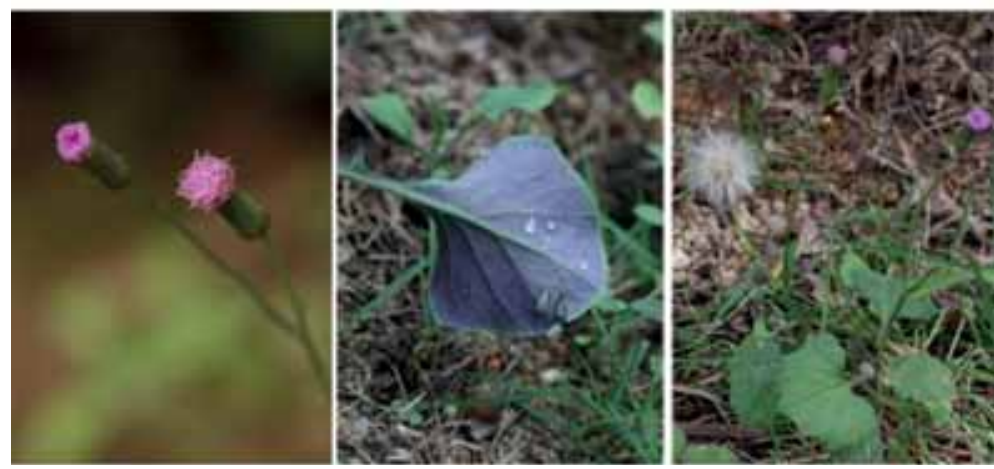

FIG I. The four types of poisonous plants causing severe toxicity in our patients (reproduced from reference 24 with permission)
Most of the patients experienced mild ( $n=49$, $79 \%)$ or moderate toxicity $(n=8,13 \%)$. Of them, 55 patients recovered within days with supportive treatment. The other two patients with nephrotoxicity had residual renal impairment after discontinuation of plant use; in one of them a renal biopsy study showed tubulonephritic changes. The remaining five patients $(8 \%)$ experienced severe toxicity after the use of Abrus precatorius ( $\mathrm{n}=1$ ), Gelsemium elegans $(\mathrm{n}=2)$, Rhododendron species $(\mathrm{n}=1)$, and Emilia sonchifolia $(\mathrm{n}=1)$, and all five required intensive care support.

The morphological identification and biochemical analysis process followed to identify the plants involved in the 62 cases of intoxication is shown in Figure 2. Most patients provided fresh plant specimens $(n=53,85 \%)$ or photograph of the plant $(n=3,5 \%)$ they had consumed. Among these specimens received by our laboratory, 43 could be identified morphologically with the aid of the Hong Kong Herbarium, Agriculture, Fisheries and Conservation Department. Biochemical analysis for specific plant toxin(s) were attempted in the plant or the biological specimens in 45 (73\%) cases, with 41 yielding diagnostic information, including six cases with no plant specimens for identification and 13 cases with unsuccessful identification. There were 22 (35\%) cases with both informative morphological identification and biochemical results. Among these 22 cases, the morphological identification and biochemical results were coherent in 20. For the remaining two cases with clinical gelsemium poisoning, gelsemium alkaloids were detected in both the urine and plant specimens. However, the plant specimen was morphologically identified as Cassytha filiformis. Cassytha filiformis is a non-toxic plant that has been shown to parasitise Gelsemium elegans and absorb gelsemium alkaloids, leading to poisoning. ${ }^{18}$ Patient accuracy in identification of plants was low. Although 56 (90\%) patients had given common names of the plants they consumed, only 19 of them were correct.

Patients consumed the plants as medicinal herbs $(n=31,50 \%)$, as food $(n=29,47 \%)$, in an attempted suicide $(n=1,2 \%)$, or accidentally, in one paediatric patient $(n=1,2 \%)$. Causes of poisoning in patients using the plants as medicinal herbs or food included plant misidentification $(n=34)$, unawareness/underestimation of the potential toxicity $(n=20)$, or contamination of non-toxic plants with poisonous ones $(n=6)$. The sources of the poisonous plants used included self-collecting from parks or the countryside $(n=37,60 \%)$, obtaining from friends or relatives $(n=20,32 \%)$, growing at home $(n=3,5 \%)$, and buying from wet markets $(n=2$, $3 \%)$. The plants were obtained in Hong Kong $(n=49$, $79 \%)$ or mainland China $(n=12,19 \%)$, with one $(2 \%)$ case collected from the Philippines. 


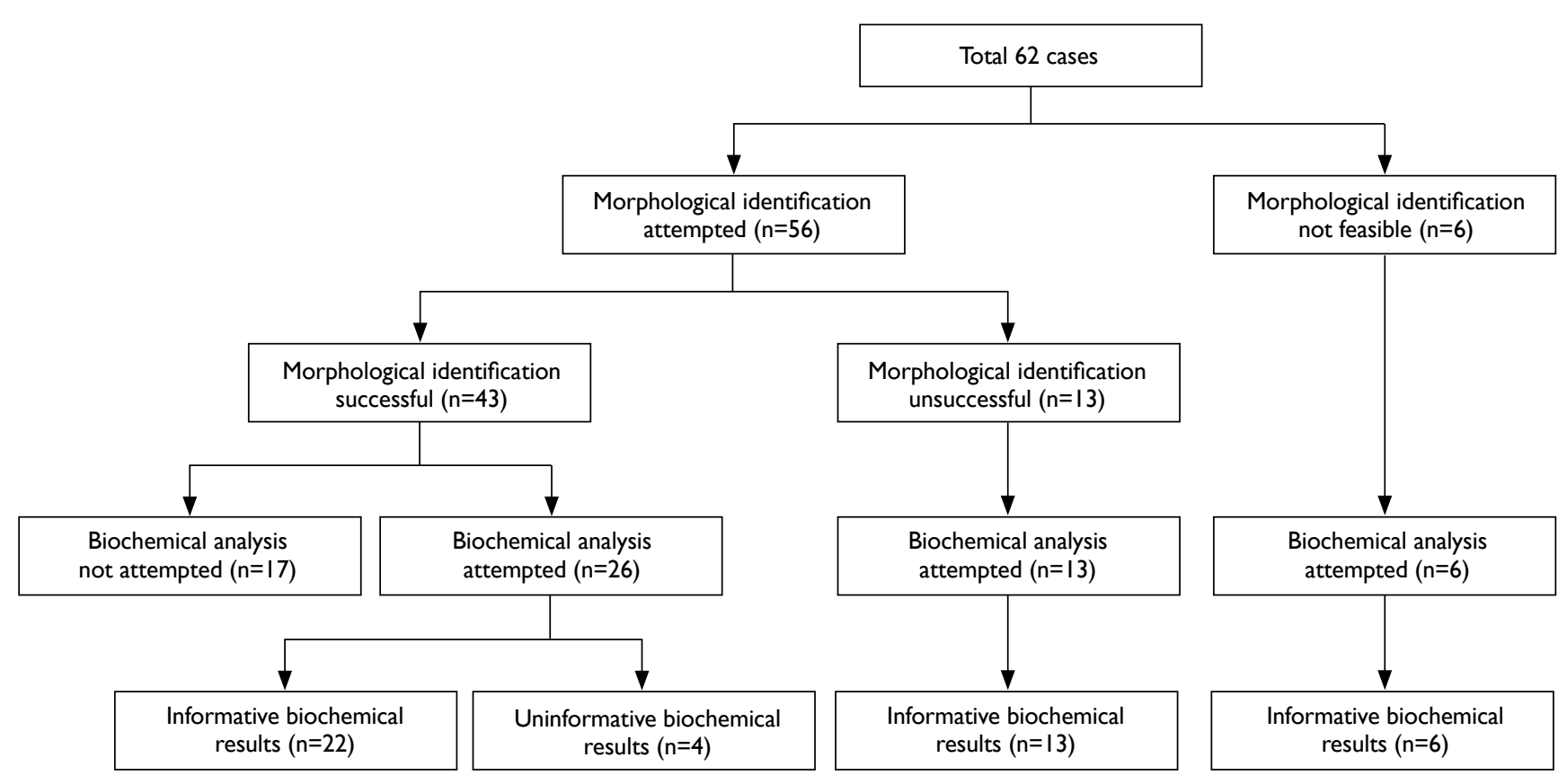

FIG 2. Flowchart showing morphological identification and biochemical analysis performed in the 62 cases of plant intoxication in this study

\section{Discussion}

The 62 cases reported herein represent the largest series of plant poisoning cases in Hong Kong confirmed by either morphological identification or biochemical confirmation. Comparing our results with those of studies from other regions, the pattern of plant poisoning is considerably different. Plant poisonings reported in Western countries are predominantly accidental exposure in children, ${ }^{3,7,10}$ whereas in the present study, most patients were adults poisoned after intentional use of wild plants. The highly urbanised and industrialised nature of Hong Kong explains the low incidence of paediatric accidental poisoning. The relatively high rate of adult poisoning may be related to the long-standing tradition and Chinese culture of using wild plant as "medicinal herbs" and "vegetables". Recreational abuse of toxic plants, such as Datura species, ${ }^{26,27}$ which are responsible for a significant number of poisoning cases reported in other regions, was not observed locally.

The most common type of poisoning in the current study was the use of raphide-containing plants, such as Alocasia macrorrhizos, accounting for more than $25 \%$ of cases. The roots of these plants are frequently mistaken as edible taro. ${ }^{28}$ Although all cases in this study were mild, severe outcomes including oropharyngeal oedema, upper airway obstruction, or systemic toxicity are possible..$^{29,30}$
Similar to previous reports of plant poisoning, ${ }^{3,5,7}$ most patients in the present study developed mild transient symptoms and recovered with supportive management and discontinuation of exposure. However, there were five cases of severe poisoning requiring intensive care support, involving the use of Gelsemium elegans, Rhododendron species, Abrus precatorius, and Emilia sonchifolia (Fig 1).

Gelsemium elegans is one of the most notorious poisonous plants in Hong Kong and South-East Asian countries and has been used for homicidal and suicidal purposes. ${ }^{31,32}$ Severe gelsemium toxicity can result in respiratory depression and even death. In our case series, two clusters of "hidden" gelsemium poisoning were identified where the patients consumed non-toxic parasitic Cassytha filiformis which absorbed gelsemium alkaloids from Gelsemium elegans on which it grew. ${ }^{18}$ Similar cases of hidden gelsemium poisoning from contaminated dried herbs Ficus hirta have been reported in Hong Kong. ${ }^{16}$

Rhododendron species and other plants in the Ericaceae family contain grayanotoxins. ${ }^{33}$ Severe grayanotoxin poisoning may result in respiratory depression and arrhythmias. ${ }^{34}$ "Mad honey" containing nectar of Ericaceae plants is known to be a source of exposure in Hong Kong, ${ }^{35}$ but poisonings due to direct consumption of Rhododendron flowers are not uncommon. ${ }^{12}$ 
The seed of Abrus precatorius contains the protein abrin, an extremely poisonous toxin similar to ricin that can result in multi-organ failure. ${ }^{36,37}$ These seeds are sometimes used in beaded jewellery, in addition to being collected, providing another potential source of exposure. ${ }^{37}$ Cases of abrin poisoning due to suicidal attempt or accidental ingestion have been reported worldwide, ${ }^{38-40}$ but such poisoning is rare in Hong Kong.

The toxic constituents in Emilia sonchifolia are pyrrolizidine alkaloids. ${ }^{41}$ Massive acute ingestion can lead to hepatotoxicity and coagulopathy, while chronic low-dose exposure can result in liver cirrhosis and hepatic veno-occlusive disease. ${ }^{42-44}$ Certain types of traditional Chinese medicine, such as Flos farfarae and Herba senecionis scandentis, are potential sources of pyrrolizidine alkaloids exposure in Hong Kong, ${ }^{45,46}$ but toxicity due to wild plant consumption is relatively rare. ${ }^{14}$

Timely diagnosis of plant poisonings is very difficult. Local epidemiology data are scarce, and reports published in other parts of the world are of limited use because plant species are geographically specific. Patients might volunteer a history of plant exposure, but the information they provide may be uninformative, misleading, or incorrect. In our case series, among those who provided a common name of the plant, only $34 \%$ were correct.

Our results demonstrate the importance of adopting a complementary approach, incorporating clinical toxidrome, morphological identification of plant specimens, and biochemical analysis of plant toxins, in order to achieve maximal diagnostic efficacy. Clinical history may not be particularly informative. The presenting toxidrome is more objective and useful for identifying the plant responsible for the poisoning, and this diagnostic approach is well supported in the literature. ${ }^{20,21}$

Table 1 summarises and categorises commonly encountered local poisonous plants by toxidromes. This may serve as quick reference for clinicians to allow rapid identification of the plant and prompt management of the poisoning. However, clinicians should also be aware that the toxicity of some plants may not be well characterised, and that some plants may give rise to non-specific toxidrome, rendering this approach less useful in certain cases.

Specialised toxicological investigation may provide additional evidence to confirm or refute the provisional diagnosis. In most situations, morphological identification of the plant is sufficient to achieve a diagnosis, if a well-preserved plant specimen is provided. However, a plant specimen is not always available; even if available, it may not be representative of the plants consumed by the patient, or may have been deformed due to prior processing. An equally important tool is target testing for specific plant toxin(s) in the plant or biological specimens based on clinical suspicion. This is a powerful tool for confirming the diagnosis, especially when plant specimen is not available; and for identifying cases of contamination with an unclear or hidden source. ${ }^{18}$ However, these investigations cannot aid immediate management in the emergency room.

Diagnosis of plant poisoning requires a proactive rather than a reactive approach. A knowledge database comprising the clinical toxicity, morphology, and biochemical analysis of local toxic plants is an important tool to prepare clinicians and healthcare professionals, such as that provided by the Hospital Authority. ${ }^{24}$ This public resource can also be used to educate the general public on the dangers of wild plants.

As a retrospective study, this study has several limitations. This study is subjected to selection bias as the data collection is a passive procedure depending on test or consultation requests by clinicians, and there might be incomplete or missing data. Moreover, for those with history of exposure or mild clinical symptoms, toxicology testing or consultation may not be requested, our data might therefore include only cases with more severe outcome in the overall continuum of plant poisoning. Inevitably, some plant exposures were not reported to our laboratory, and thus our findings may underestimate the actual number of cases.

\section{Conclusions}

Diagnosing plant poisoning is challenging and the epidemiology of plant poisonings is geographically specific. Clinicians should consider a complementary approach with consideration of the clinical toxidromes, local epidemiological data, botanical and toxicological findings to help recognition of the plants involved in cases of intoxication. Plant specimens and biological specimens should be saved whenever possible for toxicological analysis. Clinicians should be aware of local poisonous plants and their toxicities. Although most plant exposure resulted in a self-limiting disease, severe poisonings were encountered. The public should be educated about the potential hazards of consuming plants obtained from the wild and discouraged from engaging in this practice.

\section{Author contributions}

All authors had full access to the data, contributed to the study (including concept or design, acquisition of data, analysis or interpretation of data, drafting of the manuscript, and critical revision for important intellectual content), approved the final version for publication, and take responsibility for its accuracy and integrity.

\section{Conflicts of interest}

All authors have disclosed no conflicts of interest. 


\section{Declaration}

Some of the cases have been published by the authors in electronic form ${ }^{24}$ and in book form (Mak WL, Lam YH, Ching CK, Chan SS, Chong YK, Ng WY, editors. Atlas of Poisonous Plants in Hong Kong-A Clinical Toxicology Perspective. Hong Kong: Hospital Authority Toxicology Reference Laboratory; 2016), and presented at the Hospital Authority Toxicology Services Scientific Conference 2016 ("Poisonous plants in Hong Kong-a clinical perspective"). Some of the cases have been previously published as case reports by the authors and other units. ${ }^{12-19}$

\section{Funding/support}

This research received no specific grant from any funding agency in the public, commercial, or not-for-profit sectors.

\section{Ethics approval}

The study was approved by the Hong Kong Hospital Authority Kowloon West Cluster Research Ethics Committee (Ref. KW/ EX-16-036[96-18(TCM)]).

\section{References}

1. Children's Health Queensland Hospital and Health Service, Queensland Government. Queensland Poisons Information Centre Annual report 2014. Available from: https://www.childrens.health.qld.gov.au/wp-content/ uploads/PDF/poisons/annual-reports/2014.pdf. Accessed 30 May 2018.

2. Müller D, Desel H. Common causes of poisoning: etiology, diagnosis and treatment. Dtsch Arztebl Int 2013;110:6909.

3. Plenert B, Prasa D, Hentschel H, Deters M. Plant exposures reported to the Poisons Information Centre Erfurt from 2001-2010. Planta Med 2012;78:401-8.

4. Rhalem N, Chaoui H, Lahcen O, Soulaymani A, Bencheikh RS. Toxic deaths: data from the Poison Control Centre of Morocco (CAPM). XXXV International Congress of the European Association of Poisons Centres and Clinical Toxicologists (EAPCCT) 26-29 May 2015, St Julian's, Malta. Clin Toxicol 2015;53:265.

5. Slaughter RJ, Beasley DM, Lambie BS, Wilkins GT, Schep LJ. Poisonous plants in New Zealand: a review of those that are most commonly enquired about to the National Poisons Centre. N Z Med J 2012;125:87-118.

6. Swedish Poisons Information Centre. Swedish Poisons Information Centre. Annual Report 2015. Available from: https://giftinformation.se/globalassets/publikationer/ annual-report-2015.pdf. Accessed 18 May 2018.

7. Sriapha C, Tongpoo A, Wongvisavakorn S, et al. Plant poisoning in Thailand: a 10-year analysis from Ramathibodi Poison Center. Southeast Asian J Trop Med Public Health 2015;46:1063-76.

8. Public Health England. National Poisons Information Service Report 2016/17. Available from: http://www. edinburghclinicaltoxicology.org/s/NPIS-2016-17-report. pdf. Accessed 30 May 2018.

9. Gummin DD, Mowry JB, Spyker DA, Brooks DE, Fraser MO, Banner W. 2016 annual report of the American Association of Poison Control Centers' National Poison Data System (NPDS): 34th annual report. Clinical Toxicol (Phila) 2017;55:1072-252.

10. Enfield B, Brooks DE, Welch S, et al. Human plant exposures reported to a regional (Southwestern) Poison Control Center over 8 years. J Med Toxicol 2018;14:74-8.

11. Chan TY, Chan LY, Tam LS, Critchley JA. Neurotoxicity following the ingestion of a Chinese medicinal plant, Alocasia macrorrhiza. Hum Exp Toxicol 1995;14:727-8.

12. Poon WT, Ho CH, Yip KL, et al. Grayanotoxin poisoning from Rhododendron simsii in an infant. Hong Kong Med J 2008;14:405-7.

13. Poon WT, Chau TL, Lai CK, et al. Hepatitis induced by Teucrium viscidum. Clin Toxicol (Phila) 2008;46:819-22.

14. Wu JS, Poon WT, Ma CK, et al. Budd-Chiari syndrome secondary to toxic pyrrolizidine alkaloid exposure. Hong Kong Med J 2013;19:553-5.

15. Pang CT, Ng HW, Lau FL. Oral mucosal irritating plant ingestion in Hong Kong: epidemiology and its clinical presentation. Hong Kong J Emerg Med 2010;17:477-81.

16. Lai CK, Chan YW. Confirmation of gelsemium poisoning by targeted analysis of toxic gelsemium alkaloids in urine. J Anal Toxicol 2009;33:56-61.

17. Fung HT, Lam KK, Lam SK, Wong OF, Kam CW. Two cases of Gelsemium elegans Benth. poisoning. Hong Kong J Emerg Med 2007;14:221-4.

18. Cheung WL, Law CY, Lee HC, et al. Gelsemium poisoning mediated by the non-toxic plant Cassytha filiformis parasitizing Gelsemium elegans. Toxicon 2018;154:42-9.

19. Cheng KL, Chan YC, Mak TW, Tse ML, Lau FL. Chinese herbal medicine-induced anticholinergic poisoning in Hong Kong. Hong Kong Med J 2013;19:38-41.

20. Diaz JH. Poisoning by herbs or plants: rapid toxidromic classification and diagnosis. Wilderness Environ Med 2016;27:136-52.

21. Lin TJ, Nelson LS, Tsai JL, et al. Common toxidromes of plant poisonings in Taiwan. Clin Toxicol (Phila) 2009;47:161-8.

22. Ng SW, Ching CK, Chan AY, Mak TW. Simultaneous detection of 22 toxic plant alkaloids (aconitum alkaloids, solanaceous tropane alkaloids, sophora alkaloids, strychnos alkaloids and colchicine) in human urine and herbal samples using liquid chromatography-tandem mass spectrometry. J Chromatogr B Analyt Technol Biomed Life Sci 2013;942-943:63-9.

23. Persson HE, Sjöberg GK, Haines JA, Pronczuk de Garbino J. Poisoning severity score. Grading of acute poisoning. J Toxicol Clin Toxicol 1998;36:205-13.

24. Hospital Authority Toxicology Reference Laboratory. Atlas of poisonous plants in Hong Kong-a clinical toxicology perspective. 2016. Available from: http://www3.ha.org.hk/ toxicplant. Accessed 30 May 2018.

25. von Elm E, Altman DG, Egger M, Pocock SJ, Gøtzsche PC, Vandenbroucke JP; STROBE Initiative. The Strengthening the Reporting of Observational Studies in Epidemiology (STROBE) statement: guidelines for reporting observational studies. J Clin Epidemiol 2008;61:344-9.

26. Centers for Disease Control and Prevention (CDC). Suspected moonflower intoxication-Ohio, 2002. MMWR Morb Mortal Wkly Rep 2003;52:788-91.

27. Boumba VA, Mitselou A, Vougiouklakis T. Fatal poisoning from ingestion of Datura stramonium seeds. Vet Hum Toxicol 2004;46:81-2.

28. Lin TJ, Hung DZ, Hu WH, Yang DY, Wu TC, Deng JF. Calcium oxalate is the main toxic component in clinical presentations of Alocasis macrorrhiza (L) Schott and Endl poisonings. Vet Hum Toxicol 1998;40:93-5. 
29. Farré M, Xirgu J, Salgado A, Peracaula R, Reig R, Sanz P. Fatal oxalic acid poisoning from sorrel soup. Lancet 1989;2:1524.

30. Kalliala H, Kauste O. Ingestion of rhubarb leaves as cause of oxalic acid poisoning. Ann Paediatr Fenn 1964;10:22831.

31. Xiang H, Zhou YJ, Huang PL, et al. Lethal poisoning with Gelsemium elegans in Guizhou, China. Public Health 2016;136:185-7.

32. Zhou Z, Wu L, Zhong Y, et al. Gelsemium elegans poisoning: a case with 8 months of follow-up and review of the literature. Front Neurol 2017;8:204.

33. Lampe KF. Rhododendrons, mountain laurel, and mad honey. JAMA 1988;259:2009.

34. Lennerz C, Jilek C, Semmler V, Deisenhofer I, Kolb C. Sinus arrest from mad honey disease. Ann Intern Med 2012;157:755-6.

35. Chen SP, Lam YH, Ng VC, et al. Mad honey poisoning mimicking acute myocardial infarction. Hong Kong Med J 2013;19:354-6.

36. Dickers KJ, Bradberry SM, Rice P, Griffiths GD, Vale JA. Abrin poisoning. Toxicol Rev 2003;22:137-42.

37. Fernando C. Poisoning due to Abrus precatorius (jequirty bean). Anaesthesia 2001;56:1178-80.

38. Wooten JV, Pittman CT, Blake TA, et al. A case of abrin toxin poisoning, confirmed via quantitation of L-abrine (N-methyl-L-tryptophan) biomarker. J Med Toxicol 2014;10:392-4.
39. Jang DH, Hoffman RS, Nelson LS. Attempted suicide, by mail order: Abrus precatorius. J Med Toxicol 2010;6:42730.

40. Subrahmanyan D, Mathew J, Raj M. An unusual manifestation of Abrus precatorius poisoning: a report of two cases. Clin Toxicol (Phila) 2008;46:173-5.

41. Cheng D, Röder E. Pyrrolizidine alkaloids from Emilia sonchifolia [in German]. Planta Med 1986;6:484-6.

42. Wiedenfeld $H$. Plants containing pyrrolizidine alkaloids: toxicity and problems. Food Addit Contam Part A Chem Anal Control Expo Risk Assess 2011;28:282-92.

43. Chen Z, Huo JR. Hepatic veno-occlusive disease associated with toxicity of pyrrolizidine alkaloids in herbal preparations. Neth J Med 2010;68:252-60.

44. Kumana CR, Ng M, Lin HJ, Ko W, Wu PC, Todd D. Herbal tea induced hepatic veno-occlusive disease: quantification of toxic alkaloid exposure in adults. Gut 1985;26:101-4.

45. Centre for Food Safety, Food and Environmental Hygiene Department, Hong Kong SAR Government. Risk assessment studies report no. 56. Chemical hazard evaluation. Pyrrolizidine alkaloids in food. 2017. Available from: https://www.cfs.gov.hk/english/programme/ programme_rafs/files/Pyrrolizidine_Alkaloids_in_Food_e. pdf. Accessed 10 Nov 2018.

46. Gao H, Ruan JQ, Chen J, et al. Blood pyrrole-protein adducts as a diagnostic and prognostic index in pyrrolizidine alkaloid-hepatic sinusoidal obstruction syndrome. Drug Des Devel Ther 2015;9:4861-8. 